\title{
Risk factors for PICU admission and death among children and young people hospitalized with COVID-19 and PIMS-TS in England during the first pandemic year
}

\author{
Joseph L. Ward ${ }^{1 凶}$, Rachel Harwood ${ }^{2,3}$, Clare Smith ${ }^{(1)}{ }^{4}$, Simon Kenny ${ }^{2,3,4}$, Matthew Clark (i]) 4 , \\ Peter J. Davis $\mathbb{1}^{4,5}$, Elizabeth S. Draper ${ }^{6}$, Dougal Hargreaves $\mathbb{D}^{7}$, Shamez Ladhani ${ }^{8}$, Michael Linney ${ }^{9}$, \\ Karen Luyt ${ }^{10}{ }^{10}$, Steve Turner ${ }^{11,12}$, Elizabeth Whittaker ${ }^{10}{ }^{13,14}$, Lorna K. Fraser ${ }^{15}{ }^{15}$ and Russell M. Viner (iD)
}

Identifying which children and young people (CYP) are most vulnerable to serious infection due to severe acute respiratory syndrome coronavirus 2 (SARS-CoV-2) is important to guide protective interventions. To address this question, we used data for all hospitalizations in England among 0-17 year olds from 1 February 2019 to 31 January 2021. We examined how sociodemographic factors and comorbidities might be risk factors for pediatric intensive care unit (PICU) admission among hospitalizations due to the following causes: Coronavirus Disease 2019 (COVID-19) and pediatric inflammatory multi-system syndrome temporally associated with SARS-CoV-2 (PIMS-TS) in the first pandemic year (2020-2021); hospitalizations due to all other non-traumatic causes in 2020-2021; hospitalizations due to all non-traumatic causes in 2019-2020; and hospitalizations due to influenza in 2019-2020. Risk of PICU admission and death from COVID-19 or PIMS-TS in CYP was very low. We identified 6,338 hospitalizations with COVID-19, of which 259 were admitted to a PICU and eight CYP died. We identified 712 hospitalizations with PIMS-TS, of which 312 were admitted to a PICU and fewer than five CYP died. Hospitalizations with COVID-19 and PIMS-TS were more common among males, older CYP, those from socioeconomically deprived neighborhoods and those who were of non-White ethnicity (Black, Asian, Mixed or Other). The odds of PICU admission were increased in CYP younger than 1 month old and decreased among 15-17 year olds compared to 1-4 year olds with COVID-19; increased in older CYP and females with PIMS-TS; and increased for Black compared to White ethnicity in patients with COVID-19 and PIMS-TS. Odds of PICU admission in COVID-19 were increased for CYP with comorbidities and highest for CYP with multiple medical problems. Increases in odds of PICU admission associated with different comorbidities in COVID-19 showed a similar pattern to other causes of hospitalization examined and, thus, likely reflect background vulnerabilities. These findings identify distinct risk factors associated with PICU admission among CYP with COVID-19 or PIMS-TS that might aid treatment and prevention strategies.

M ost CYP experience a mild disease after SARS-CoV-2 infection compared to adults ${ }^{1-3}$, and asymptomatic infection is common ${ }^{4}$. However, severe clinical outcomes have been reported in CYP due to COVID-19 and to PIMS-TS or multi-system inflammatory syndrome in children (MIS-C), including a small number of deaths $s^{2,5-9}$. Understanding which CYP are vulnerable to increased risk is important to guide clinicians, families and policymakers in relation to protective shielding and potential vaccination strategies.

Early in the pandemic, guidance from the UK Royal College of Paediatrics and Child Health identified CYP with immunodeficiency or immunosuppression and those with certain malignancies as having the greatest vulnerability to COVID-19 ${ }^{10}$. However, CYP with a broad range of other conditions have also been highlighted as being potentially clinically extremely vulnerable (CEV). CYP in many countries who are identified as CEV have been advised to take additional 'shielding' precautions to reduce the risk of SARS-CoV-2 infection. These include measures that may result in harm to CYP and their families, including measures associated with reduced social mixing and restriction of inperson schooling.

To limit harms due to inappropriate shielding, clear guidance is urgently needed on which CYP are at higher risk of poorer outcomes of SARS-CoV-2 infection. The rarity of severe and fatal COVID-19 in CYP means that large-scale, population-based studies are needed to identify CYP at greatest risk. These analyses also need to take into account background risks for severe illness that preceded the pandemic. CYP who are at increased risk of severe disease due to

'UCL Great Ormond St. Institute of Child Health, London, UK. Institute of Systems, Molecular and Integrative Biology, University of Liverpool, Liverpool, UK. ${ }^{3}$ Dept of Paediatric Surgery, Alder Hey Children's NHS Foundation Trust, Liverpool, UK. ${ }^{4}$ NHS England and Improvement, Leeds, UK. ${ }^{5}$ Paediatric Intensive Care Unit, Bristol Royal Hospital for Children, Bristol, UK. ${ }^{6}$ PICANet, Department of Health Sciences, University of Leicester, Leicester, UK. ${ }^{7}$ Mohn Centre for Children's Health and Wellbeing, Imperial College London, London, UK. ${ }^{8}$ Immunisation and Countermeasures, Public Health England, London, UK. ' University Hospitals Sussex NHS Foundation Trust, Brighton, UK. ${ }^{10}$ National Child Mortality Database, Bristol Medical School, University of Bristol, Bristol, UK. "NHS Grampian, Aberdeen, UK. ${ }^{12}$ University of Aberdeen, Aberdeen, UK. ${ }^{13}$ Paediatric Infectious Diseases, Imperial College Healthcare NHS Trust, London, UK. ${ }^{14}$ Section of Paediatric Infectious diseases, Imperial College London, London, UK. ${ }^{15}$ Martin House Research Centre, Dept of Health Sciences, University of York, York, UK. 『e-mail: joseph.ward@ucl.ac.uk 
Table 1 | Number and proportion of admissions by sociodemographic characteristics within each cohort (COVID-19, PIMS-TS, other pandemic year admissions, all admissions in 2019-2020, influenza admissions in 2019-2020)

\begin{tabular}{|c|c|c|c|c|c|c|c|c|c|c|c|}
\hline & \multicolumn{6}{|c|}{$2020-2021$} & \multicolumn{4}{|c|}{ 2019-2020 } \\
\hline & & \multicolumn{2}{|c|}{ COVID-19 } & \multicolumn{2}{|c|}{ PIMS-TS } & \multicolumn{2}{|c|}{$\begin{array}{c}\text { Other pandemic year } \\
\text { admissions }\end{array}$} & \multicolumn{2}{|c|}{$\begin{array}{l}\text { All admissions } \\
\text { 2019-2020 }\end{array}$} & \multicolumn{2}{|c|}{ Influenza 2019-2020 } \\
\hline & & $n$ & $(\%)$ & $n$ & $(\%)$ & $n$ & $(\%)$ & $n$ & $(\%)$ & $n$ & $(\%)$ \\
\hline Total & & 6,338 & $(100.0)$ & 712 & $(100.0)$ & 463,556 & $(100.0)$ & 771,591 & $(100.0)$ & 6,968 & $(100.0)$ \\
\hline \multirow[t]{2}{*}{ Sex } & Male & 3,347 & $(52.8)$ & 452 & $(63.5)$ & 247,299 & $(53.3)$ & 416,830 & $(54.0)$ & 3,733 & $(53.6)$ \\
\hline & Female & 2,991 & $(47.2)$ & 260 & $(36.5)$ & 216,257 & $(46.7)$ & 354,761 & $(46.0)$ & 3,235 & $(46.4)$ \\
\hline & Post-neonatal & 1,216 & $(19.2)$ & 71 & $(10.0)$ & 71,560 & $(15.4)$ & 135,195 & $(17.5)$ & 1,036 & $(14.9)$ \\
\hline & $1-4$ & 1,281 & $(20.2)$ & 217 & $(30.5)$ & 126,426 & $(27.3)$ & 262,511 & $(34.0)$ & 3,189 & $(45.8)$ \\
\hline & $5-9$ & 840 & (13.3) & 216 & (30.3) & 71,255 & $(15.4)$ & 116,951 & $(15.2)$ & 1,274 & $(18.3)$ \\
\hline & $10-14$ & 1,188 & $(18.7)$ & 175 & $(24.6)$ & 72,256 & $(15.6)$ & 97,662 & $(12.7)$ & 871 & $(12.5)$ \\
\hline & $15-17$ & 1,072 & $(16.9)$ & 31 & $(4.4)$ & 52,829 & $(11.4)$ & 69,450 & $(9.0)$ & 447 & $(6.4)$ \\
\hline & Black & 395 & $(6.2)$ & 109 & $(15.3)$ & 17,388 & $(3.8)$ & 30,948 & $(4.0)$ & 333 & $(4.8)$ \\
\hline & Other & 298 & $(4.7)$ & 40 & $(5.6)$ & 13,160 & $(2.8)$ & 22,381 & $(2.9)$ & 197 & $(2.8)$ \\
\hline & Unknown & 478 & $(7.5)$ & 72 & $(10.1)$ & 34,007 & (7.3) & 51,790 & $(6.7)$ & 463 & $(6.6)$ \\
\hline \multirow{6}{*}{$\begin{array}{l}\text { IMD } \\
\text { quintile } \\
\text { category }\end{array}$} & Most deprived & 1,662 & $(26.2)$ & 163 & $(22.9)$ & 108,096 & (23.3) & 188,391 & $(24.4)$ & 1,906 & $(27.4)$ \\
\hline & 2nd most deprived & 1,533 & $(24.2)$ & 182 & $(25.6)$ & 96,386 & $(20.8)$ & 163,405 & $(21.2)$ & 1,497 & $(21.5)$ \\
\hline & 3rd most deprived & 1,218 & $(19.2)$ & 164 & $(23.0)$ & 92,034 & $(19.9)$ & 152,297 & $(19.7)$ & 1,278 & $(18.3)$ \\
\hline & 4th most deprived & 1,087 & $(17.2)$ & 98 & $(13.8)$ & 87,873 & $(19.0)$ & 142,097 & $(18.4)$ & 1,197 & $(17.2)$ \\
\hline & Least deprived & 838 & $(13.2)$ & 105 & $(14.7)$ & 78,982 & $(17.0)$ & 125,167 & $(16.2)$ & 1,090 & $(15.6)$ \\
\hline & Missing & 0 & $(0.0)$ & 0 & $(0.0)$ & 185 & $(0.0)$ & 234 & $(0.0)$ & 0 & $(0.0)$ \\
\hline
\end{tabular}

SARS-CoV-2 infection might also be those who are vulnerable to other respiratory viruses, such as influenza ${ }^{11}$.

We used national linked administrative health data (Secondary Use Services, (SUS) data, linked with the national SARS-CoV-2 database, pediatric intensive care data and national mortality data) to analyze all hospital admissions due to COVID-19 or PIMS-TS in CYP in England from February 2020 to January 2021. Of these admissions, we examined how sociodemographic factors and pre-existing conditions recorded over the previous 5 years (from 2015-2016 to 2020-2021) were associated with odds of admission to a PICU, (which we used as a proxy for serious disease), or death. To understand if these risk factors were specific to SARS-CoV-2, represented background vulnerabilities, or reflected changes to healthcare activity caused by the pandemic, we then repeated this analysis among CYP admitted with other causes of admission that year and admissions during 2019-2020, including those admissions due to influenza.

\section{Results}

Between 1 February 2019 and 31 January 2021, there were 1,242,197 emergency non-traumatic hospital admissions in England (hereafter 'admissions') involving 892,906 CYP, and 699,397 (78\%) had only one admission. During 2020-2021, there were 470,606 admissions: 6,338 with COVID-19 among 5,830 CYP; 712 with PIMS-TS among 690 CYP; and 463,556 for other causes among 367,637 CYP. In comparison, there were 771,591 admissions among 587,115 CYP during 2019-2020, of which 6,968 were due to influenza among 6,780 CYP (Table 1 and Supplementary Material 1, Table 16. In total, 69.8\% of CYP admitted due to PIMS-TS had no prior hospital admissions compared to $49.5-54.4 \%$ of CYP in all other cohorts.
The distribution of admissions by age, sex and ethnicity differed among the COVID-19, PIMS-TS and other cohorts (Table 1). A higher proportion of the PIMS-TS cohort was male $(63.5 \%)$ compared to the other cohorts (52.8-54\%). Overall, 30.9\% of admissions with COVID-19 were in infants (children younger than 1 year, including neonates under 1 month of age and post-neonatal infants aged 1-11 months), which was similar to other pandemic year admissions and total admissions in 2019-2020 but more than for influenza (17.1\%) during this time period. Among PIMS-TS, only $10.3 \%$ of admissions were in infants, whereas more than $85 \%$ were among 1-14 year-olds. CYP with non-White ethnicity made up $41.9 \%$ of COVID-19 admissions and $60.0 \%$ of PIMS-TS admissions, which was higher than the other hospitalization cohorts we examined. There were more admissions in CYP from more deprived neighborhoods compared to least deprived in all cohorts, as assessed using Index of Multiple Deprivation (IMD) quintile category. Additional details of how IMD is determined are available in the Supplementary Material.

Among COVID-19 admissions, 53.9\% had a recorded comorbidity, and $18.0 \%$ had a life-limiting comorbidity, which was higher than for other pandemic year admissions, for all admissions in 20192020 and for influenza admissions in 2019-2020 (Supplementary Table 1). Patterns of comorbidities among admissions with PIMS-TS were different than the other cohorts, with $68.3 \%$ having any comorbidity recorded, of which $20.6 \%$ were life-limiting. Due to the multi-system nature of PIMS-TS, and of limitations in how data are recorded within SUS, many of the comorbidities recorded could have been related to complications of the disease rather than prior conditions. Although $40.2 \%$ of PIMS-TS admissions had a cardiovascular comorbidity recorded, only $5.3 \%$ had a congenital 


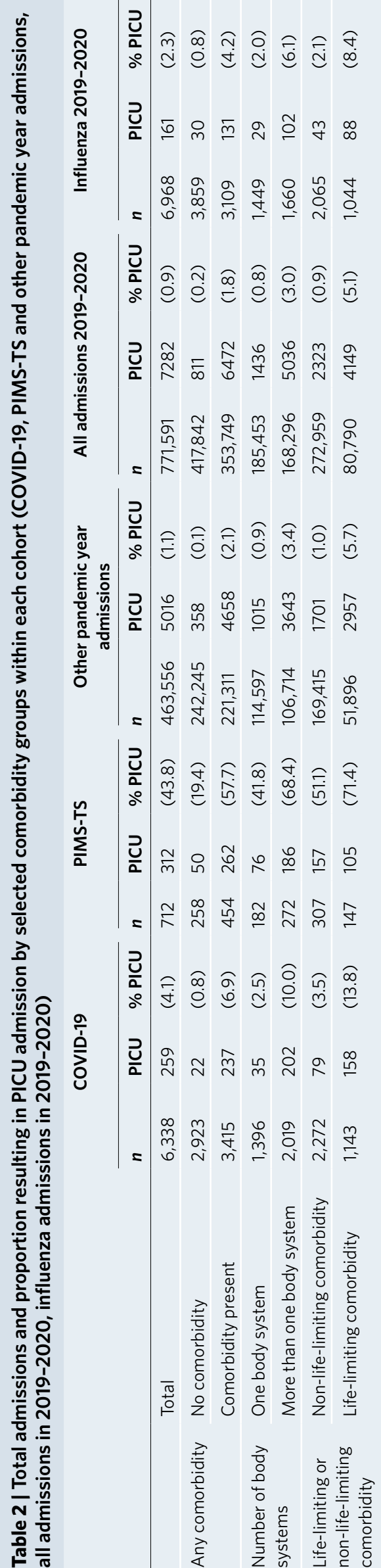

cardiac condition, with remaining codes including arrhythmias and aneurysms, which might reflect the disease process. When non-congenital cardiac conditions, blood disorders and anemias were excluded, only $15.9 \%$ of PIMS-TS admissions had a comorbidity recorded compared to $30-35 \%$ in the other cohorts.

Outcomes after admission. Table 2 shows total numbers and proportions of PICU admissions within each cohort by comorbidity category, with additional data for all comorbidities examined in Supplementary Material 1, Tables 3-5. Across COVID-19 admissions, $259(4.1 \%)$ were admitted to a PICU compared to $312(43.8 \%)$ of PIMS-TS admissions, 5,016 (1.1\%) of other pandemic year admissions, 7,282 (0.9\%) of all admissions in 2019-2020 and 161 (2.3\%) of influenza admissions in 2019-2020.

Twenty-nine CYP admitted with COVID-19 died within $28 \mathrm{~d}$ of hospitalization. Of these deaths, after reviewing case notes and death notification data, eight were confirmed as likely caused by SARS-CoV-2 infection. All had a comorbidity recorded, and seven of eight had a life-limiting condition. Six CYP died within $28 \mathrm{~d}$ of an admission with PIMS-TS, of which fewer than five were thought to be caused by the disease.

Sociodemographic factors. In multivariable models adjusting for all factors and the presence of comorbidities, female sex was associated with increased odds of PICU admission for PIMS-TS and reduced odds among all admissions in 2019-2020, with no associations found by sex for COVID-19 or the other cohorts (Supplementary Material 1, Tables 11-15). Compared to admissions among 1-4 year olds, odds of PICU admission for COVID19 were increased among neonates (CYP aged younger than 1 month) and decreased among 15-17 year olds, which was similar to patterns for other pandemic year admissions and for all admissions in 2019-2020 (although odds were also decreased for 5-14 year olds in these cohorts). Odds of PICU admission for PIMS-TS increased with age in a stepwise fashion and were highest in 15-17 year olds. The odds of PICU admission within influenza admissions in 2019-20 were only higher among neonates compared to $1-4$ year olds.

Compared to White CYP, odds of PICU admission were higher among Black CYP for COVID-19 and among Black, Asian and CYP with unknown ethnicity for PIMS-TS. Other pandemic year admissions and all admissions in 2019-2020 showed a pattern of higher odds of PICU admission in non-White ethnic groups, with no evident differences by ethnicity among influenza admissions in 2019-2020. No significant differences were observed in odds of PICU admission by IMD category for COVID-19, of all admissions in 2019-2020 and of influenza admissions in 2019-2020. By contrast, odds of PICU admission were slightly increased in less deprived categories among PIMS-TS admissions and among other pandemic year admissions.

Comorbidities. The odds of admission to a PICU were increased among CYP with any comorbidity compared to no comorbidity in all cohorts (Supplementary Material 2). The increases in odds of PICU admission associated with having each of any comorbidity, a life-limiting comorbidity or comorbidities in more than one body system for COVID-19 (Fig. 1) had overlapping confidence intervals with those for all admissions in 2019-2020 and influenza admissions in 2019-2020 but were lower than for other pandemic year admissions. Odds ratios for PIMS-TS admissions were consistently the lowest of any cohort for each comorbidity category, although confidence intervals often overlapped.

For body system comorbidities (Fig. 2), odds ratios for the increase associated with cancer/hematological conditions and neurological, respiratory, neurological with respiratory and respiratory with cardiovascular comorbidities in COVID-19 appeared to 


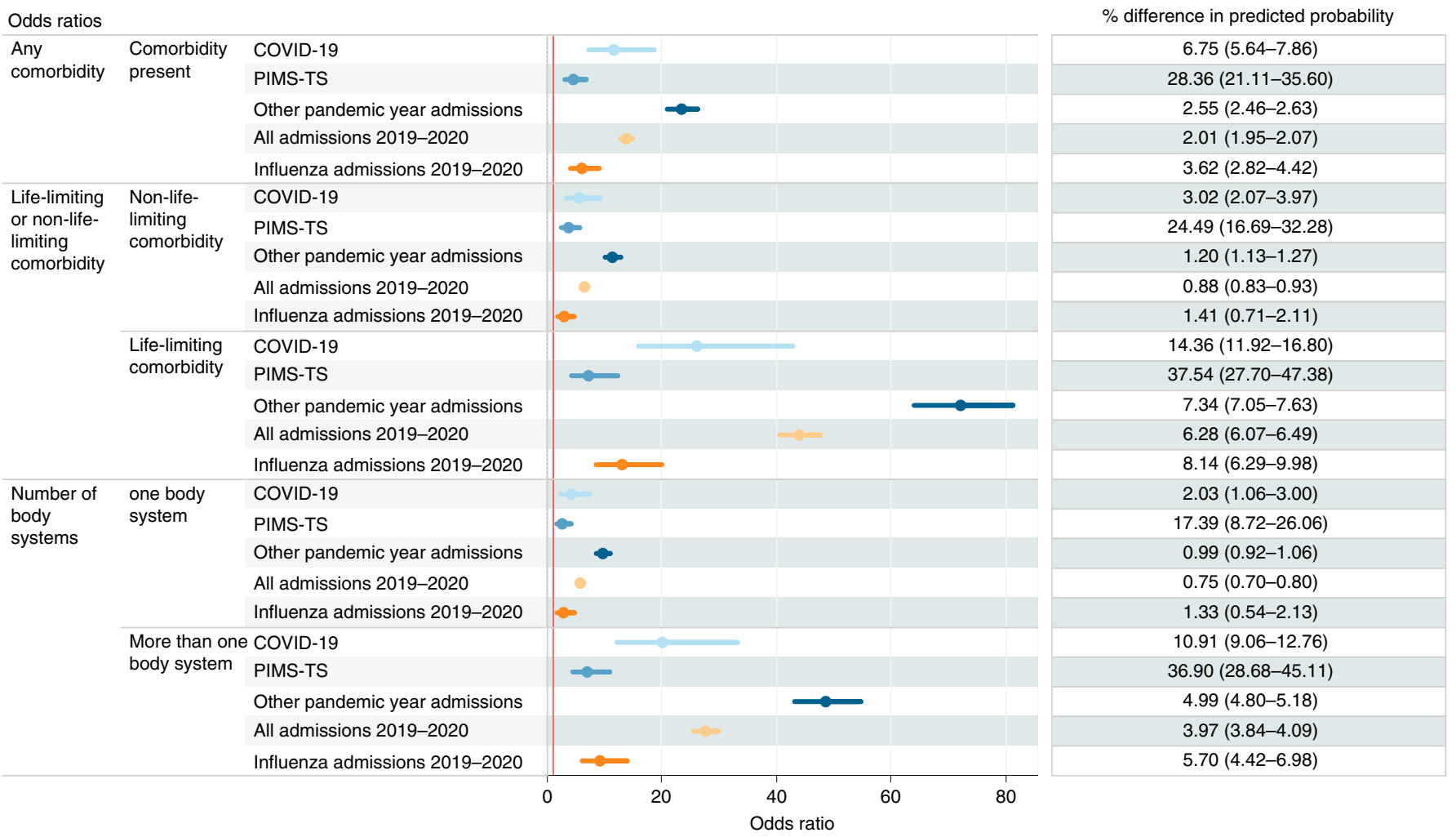

Fig. 1 | Odds ratios and percentage point difference in predicted probability with $95 \%$ confidence intervals for admission to a PICU by comorbidity groups within each cohort, adjusted for age, sex, IMD category and ethnicity. Output from GEE models. Each mark and line represents odds ratios with 95\% confidence intervals of PICU admission, within each cohort, among CYP with each comorbidity group compared to those without any comorbidity, adjusted for age, sex, IMD category and ethnicity. Percentage point difference in predicted probability of PICU admission with $95 \%$ confidence intervals are shown in the right panel. Observations: $n=6,338$ COVID-19 admissions; $n=710$ PIMS-TS admissions; $n=463,371$ other pandemic year admissions; $n=771,357$ all admissions in 2019-2020; $n=6,968$ influenza admissions in 2019-2020. These results are available in full in Supplementary Material 2.

be similar to influenza and all admissions in 2019-2020 but not PIMS-TS (where the increase in odds was lower) or other pandemic year admissions (where the increase in odds was higher). The increase in odds for cardiovascular comorbidities in COVID-19 appeared to be similar to that seen in all admissions in 2019-2020 but higher than influenza admissions in 2019-2020 and PIMS-TS and lower than for other pandemic year admissions. A similar pattern was observed for combinations of body system comorbidities (Fig. 3) -that is, that the increase in odds for COVID-19 appeared to be similar to that for influenza and all admissions in 2019-2020 but was higher than for PIMS-TS and lower than in other pandemic year admissions.

Asthma, diabetes, epilepsy and trisomy 21 each increased risk of PICU admission for COVID-19, although sickle cell disease did not (Fig. 4). Increases in odds for COVID-19 appeared broadly similar to those for other cohorts, although confidence intervals were wide, particularly for PIMS-TS.

Results from sensitivity analyses where data were restricted to 11-17 year olds to guide vaccination policy are shown in Supplementary Material 1 and 3 and Supplementary Figs. 1-4. Patterns of odds ratios were similar, although female sex was associated with significantly reduced odds of PICU admission for COVID-19. Increases in odds of PICU admission associated with comorbidities for COVID-19 among 11-17 year olds were lower than when all CYP were included for some outcomes. However, due to low numbers, confidence intervals around these estimates were wide. Also due to low numbers, we were not able to model associations within influenza admissions in 11-17 year olds.

\section{Discussion}

We found that very few CYP admitted to hospital in England due to COVID-19 or PIMS-TS went on to develop severe disease or die. Of the 12.02 million 0-17 year olds in England during 2020, 1 in $2,062(n=5,830)$ was admitted to hospital due to COVID-19, and 1 in 47,903 $(n=251)$ was admitted to a PICU. This represented only $1.3 \%$ of all secondary care admissions in the pandemic year and less than $5 \%$ of non-traumatic emergency PICU admissions. Eight of these CYP died within $28 \mathrm{~d}$ of admission to hospital. For PIMS-TS, 1 in 17,425 $(n=690)$ of CYP in England was admitted to hospital; 1 in $38,911(n=309)$ was admitted to a PICU; and fewer than five CYP died. This likely represents all PIMS-TS cases nationally over the study period, as the vast majority will have required hospitalization.

CYP admitted to hospital with COVID-19 and PIMS-TS were older and more likely to be non-White than in the other cohorts examined. For COVID-19, we found that the odds of PICU admission increased among neonates compared to 1-4 year-olds and those who were of Black compared to White ethnicity, but we found no associations by deprivation. Female sex was associated with significantly lower odds of PICU admission for COVID-19 but only in sensitivity analyses where data were restricted to 11-17 year olds. For PIMS-TS, the odds of PICU admission were increased among females, older CYP and those from non-White ethnic groups.

Of the 251 CYP admitted to a PICU with COVID-19, 91\% $(n=229)$ had an underlying condition or comorbidity. The odds of PICU admission due to COVID-19 were increased in all comorbidity categories tested except sickle cell disease. We found that 


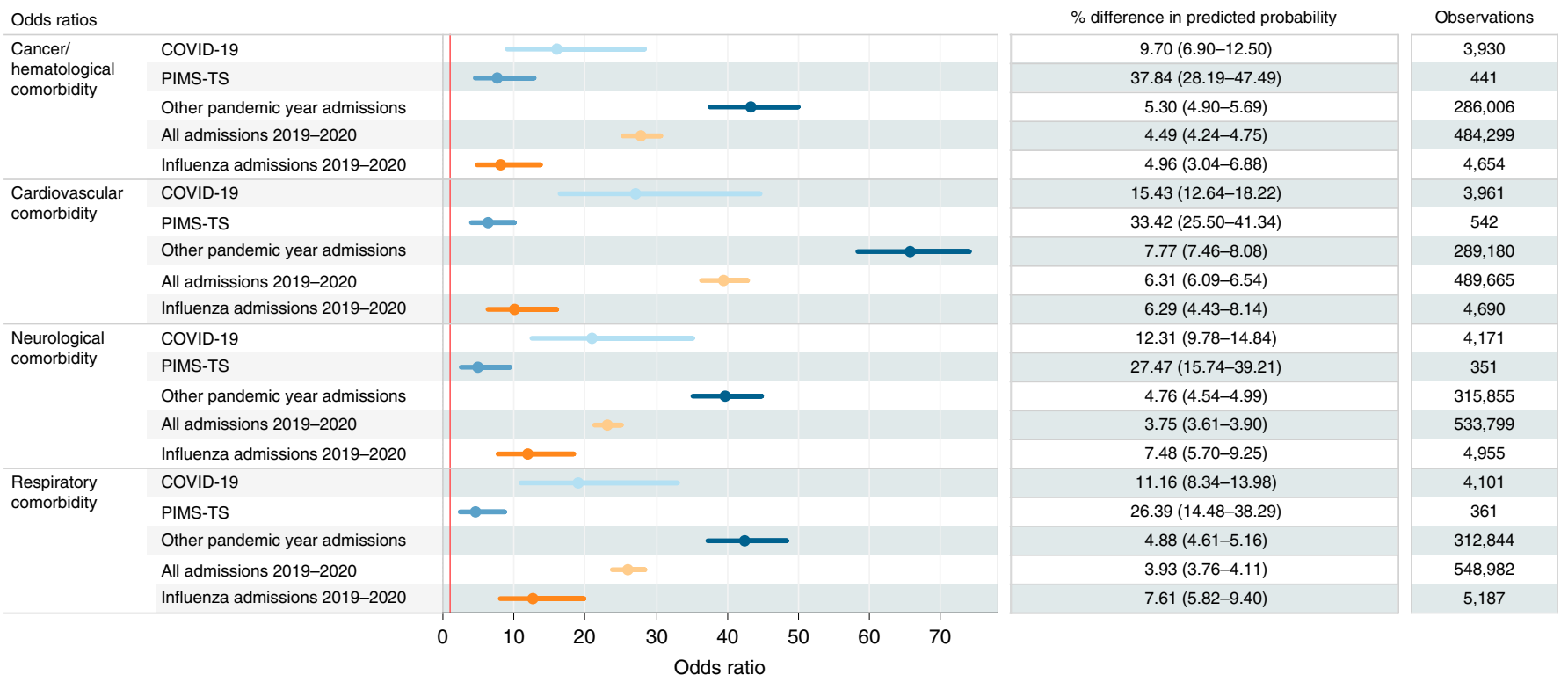

Fig. 2 | Odds ratios and percentage point difference in predicted probability with $95 \%$ confidence intervals for admission to a PICU by body system comorbidities within each cohort, adjusted for age, sex, IMD category and ethnicity. Output from GEE models. Each mark and line represents odds ratios with 95\% confidence intervals of PICU admission, within each cohort, among CYP with each comorbidity group compared to those without any comorbidity, adjusted for age, sex, IMD category and ethnicity. Percentage point difference in predicted probability of PICU admission with $95 \%$ confidence intervals are shown in the middle panel. The number of observations in each model is shown in the right panel. These results are available in full in Supplementary Material 2.

CYP with complex medical problems across multiple body systems and those with neurodisability were at greatest risk. This pattern was described in previous work $^{12}$ and is consistent with our meta-analysis of the published data, where each increase in the number of pre-existing conditions was associated with increased odds of PICU admission and death for COVID-19 ${ }^{13}$. Increases in odds of PICU admission associated with comorbidities in PIMS-TS were lower than for COVID-19 but are difficult to interpret. Coding of PIMS-TS admissions suggested that two-thirds had a comorbidity, whereas three-quarters had no prior admissions to hospital. When codes that include known cardiac and hematological complications of PIMS-TS were excluded, estimates for comorbidities in these admissions dropped to around $15 \%$, which is similar to work from the UK and the US, showing that most CYP admitted with PIMS-TS or MIS-C were previously healthy ${ }^{8,14}$.

Our comparison with other causes of admission allowed us to assess whether these risk factors are specific to COVID-19 or PIMS-TS or reflect background vulnerability to serious illness. Our findings that non-White ethnic groups (that is, CYP who were of Asian and Black ethnicity) were associated with increased odds of serious disease was similar to findings from other cohorts, except for influenza. However, a high proportion of admissions for COVID19 and PIMS-TS were from non-White ethnic groups, consistent with previous work $^{2,15,16}$, and increases in odds associated with non-White ethnicity were greater in these cohorts, which was similar to findings in adults ${ }^{17,18}$. Age patterns for COVID-19, and particularly for PIMS-TS admission, were notably shifted toward older age groups in comparison to other cohorts, including influenza. We only found significant sex differences in risk for severe COVID-19 among 11-17 year olds, unlike all admissions in 2019-2020, where female sex was associated with lower odds of PICU admission in all models. Almost two-thirds of PIMS-TS admissions were among males, which was higher than in all other cohorts, but odds of PICU admission were greater among females.

We found broadly similar increases in odds for PICU admission associated with number of body systems or type of comorbidities across COVID-19 admissions, all 2019-2020 admissions and influenza admissions. Increases in odds were highest for combinations of body system comorbidities-for example, neurological and respiratory, neurological and cardiovascular and respiratory and cardiovascular. Similarly, for the specific conditions examined, odds ratios overlapped with those for other pandemic year, all admissions in 2019-2020 and influenza, with the exception of sickle cell disease, which was not associated with an increased odds of PICU admission for COVID-19 or influenza.

When absolute risk was examined, the increases in risk associated with comorbidities were relatively small in the COVID-19, other pandemic year, all admissions in 2019-2020 and influenza cohorts, although were greater for COVID-19 than other groups. For example, for the 229 CYP with a comorbidity in one body system admitted to a PICU with COVID-19, the increase in risk above those without comorbidities was $2 \%$ for COVID-19, $0.75 \%$ for all admissions in 2019-2020 and 1.3\% for influenza. Combinations of comorbidities increased risk the most, although, again, the numbers were very small. Among the 414 admissions with respiratory and neurological comorbidities, the increase in risks were $18.3 \%$ for COVID-19 compared to $12.4 \%$ for influenza and $7-8 \%$ for other cohorts. Although this greater increase in absolute risk with COVID-19 appeared significant for body system comorbidities and their combinations, confidence intervals overlapped for all specific conditions.

Our finding that the pattern of risks for severe COVID-19 related to comorbidities is similar to that for other reasons for admission suggests that these reflect underlying vulnerabilities to illness and infection. A similar observation was made in adults when risks were examined across COVID-19 and non-COVID deaths during the pandemic ${ }^{19}$. However, although the pattern of risks was very similar, and absolute risks remained relatively small, increases in absolute risk of PICU admission were often higher for COVID19 than for other cohorts, including influenza. This suggests that SARS-CoV-2 infection might magnify underlying risks faced by CYP with chronic and life-threatening conditions. It is also possible 
Odds ratios

Neurological

and

cardiovascular

\begin{tabular}{ll} 
& All admissions 2019-2020 \\
& Influenza admissions 2019-2020 \\
\hline $\begin{array}{l}\text { Neurological } \\
\text { and respiratory }\end{array}$ & COVID-19 \\
& PIMS-TS \\
& Other pandemic year admissions \\
& All admissions 2019-2020 \\
& Influenza admissions 2019-2020 \\
\hline $\begin{array}{l}\text { Respiratory and } \\
\text { cardiovascular }\end{array}$ & COVID-19 \\
& PIMS-TS \\
& Other pandemic year admissions \\
& All admissions 2019-2020
\end{tabular}

Influenza admissions 2019-2020

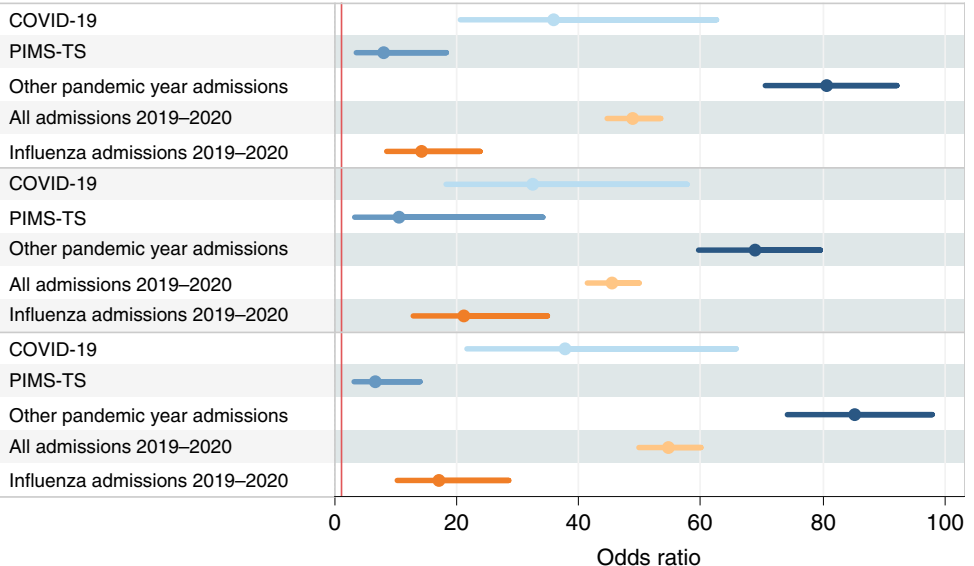

Odds ratio
$\%$ difference in predicted probability

19.34 (14.84-23.83)

$36.14(21.33-50.96)$

$9.54(9.00-10.07)$

$7.85(7.48-8.22)$

$8.69(5.75-11.64)$

$18.26(13.32-23.20)$

$39.12(18.38-59.87)$

$8.30(7.70-8.89)$

$7.28(6.90-7.66)$

$12.36(9.01-15.71)$

$20.65(15.47-25.84)$

$30.98(17.37-44.59)$

$10.06(9.44-10.68)$

$8.64(8.23-9.06)$

$10.29(7.05-13.53)$
Observations

3,456

323

265,479

454,734

4,289

3,414

288

266,108

459,563

4,368

3,357

325

261,424

450,099

4,293

Fig. 3 | Odds ratios and predicted probability with $95 \%$ confidence intervals for admission to a PICU by comorbidity combinations within each cohort, adjusted for age, sex, IMD category and ethnicity. Output from GEE models. Each mark and line represents odds ratios with $95 \%$ confidence intervals of PICU admission, within each cohort,among CYP with each comorbidity group compared to those without any comorbidity, adjusted for age, sex, IMD category and ethnicity. Percentage point difference in predicted probability of PICU admission with $95 \%$ confidence intervals are shown in the middle panel. The number of observations in each model is shown in the right panel. These results are available in full in Supplementary Material 2.

that these findings reflect changes in health system factors during the pandemic, although other studies have suggested that there was no overall change in thresholds for PICU admission in England ${ }^{20}$.

Patterns within admissions due to COVID-19 among CYP (older age, non-White ethnicity and presence of comorbidities) are very similar to those identified for adults ${ }^{17,18}$. This suggests that the strong age-related risk of severe disease in adult COVID-19 ${ }^{18,21}$ extends across the early life course, but this has previously been difficult to uncover in CYP due to the extreme rarity of severe disease.

Strengths and limitations. Previous studies examining risk factors for severe disease and death from SARS-CoV-2 in CYP predominantly used dedicated reporting systems and analyzed data in the first months of the pandemic ${ }^{2,8,9,15}$. By contrast, our study uses unique population-level data from a large country with a high burden of disease due to COVID-19 and includes all CYP admissions over the first pandemic year. We also uniquely examine data from previous years to provide context to our risk estimates. Our study is subject to several limitations. We are unable to account for the effect of protective shielding on differential exposure to SARS-Cov-2 among CYP thought to be vulnerable, which might have affected our estimates. However, our findings relate to risk factors for severe disease once hospitalized, whereas shielding is likely to bias estimates of risk factors for infection, which we did not examine.

As the pandemic progresses, and variants continue to emerge, the risks posed by SARS-CoV-2 among CYP might change. Our data included CYP infected with the Alpha variant (from November 2020 onward) but did not include CYP infected with the Delta (B.1.617.2) variant, which has been dominant in the UK since May 2021. The Delta variant has higher transmissibility and prevalence, and there have been suggestions of greater severity in CYP, although the evidence for this is mixed ${ }^{22}$. Additional population-level analyses are needed to explore the effect of this and other factors on disease severity in CYP as new data become available.

Although use of SUS data allowed us to examine the burden of severe disease associated with SARS-CoV-2 and risk factors in CYP at the population level, there are several limitations to SUS data. Missing or inaccurate data fields within SUS or other datasets and incomplete data linkage might have affected our findings. We included both cause of admission and polymerase chain reaction (PCR) testing for SARS-Cov-2 to identify CYP with COVID-19 to ensure that we captured all likely cases, but this will have affected our case definition specificity. Identifying PIMS-TS cases was particularly problematic, as International Classification of Diseases (ICD)10 codes for this condition were only introduced several months into the pandemic. We included CYP coded with Kawasaki disease and systemic inflammatory response syndrome when examining PIMS-TS, some of whom will not have had PIMS-TS (note that not all PIMS-TS cases had evidence of previous SARS-CoV-2 infection). Coding for PIMS-TS is likely to improve as knowledge of the condition increases, which will benefit future analyses of PIMS-TS admissions using hospital administrative data. There is also variation in case definition used for diagnosing post-inflammatory syndromes related to SARS-CoV-2 (for example, MIS-C and PIMS-TS), which might affect the generalizability of our results. However, in practice, the vast majority of CYP will have fulfilled both criteria ${ }^{8,15}$.

We were unable to fully distinguish between admissions with COVID-19 and admissions due to COVID-19, and some of the admissions that we classify as COVID-19 will include those with incidental positive PCR tests. We used admission to PICU as an indicator for disease severity and were not able to examine the level of intensive support needed while in critical care. Our results might also have been affected by changes to thresholds for PICU admission, as well as changes in coding practices, as the pandemic progressed and in comparison to the previous years. Our estimate for the number of deaths due to COVID-19 and PIMS-TS include only hospitalized CYP and, thus, will not include those who died at home or in an emergency department before admission. Note that our linked study of all CYP deaths up to 28 February 2021 identified 25 deaths across all places of death and provides a more complete analysis of mortality risk associated with SARS-CoV-2 ${ }^{23}$.

We use ICD-10 codes developed to identify chronic conditions across 5 years of admission data and might have missed diagnoses recorded before this. We were not able to account for the wide range of disease severity included within the diagnostic groups used for coding purposes in our analysis. Furthermore, the ICD-10 codes that we used included some diagnoses that might relate to complications of acute disease rather than pre-existing conditions only, as highlighted with PIMS-TS. We were unable to include only comorbidities before the index case to investigate this further, as many CYP had no prior records, and this approach would not account for incomplete coding in previous admissions or diagnoses made in 


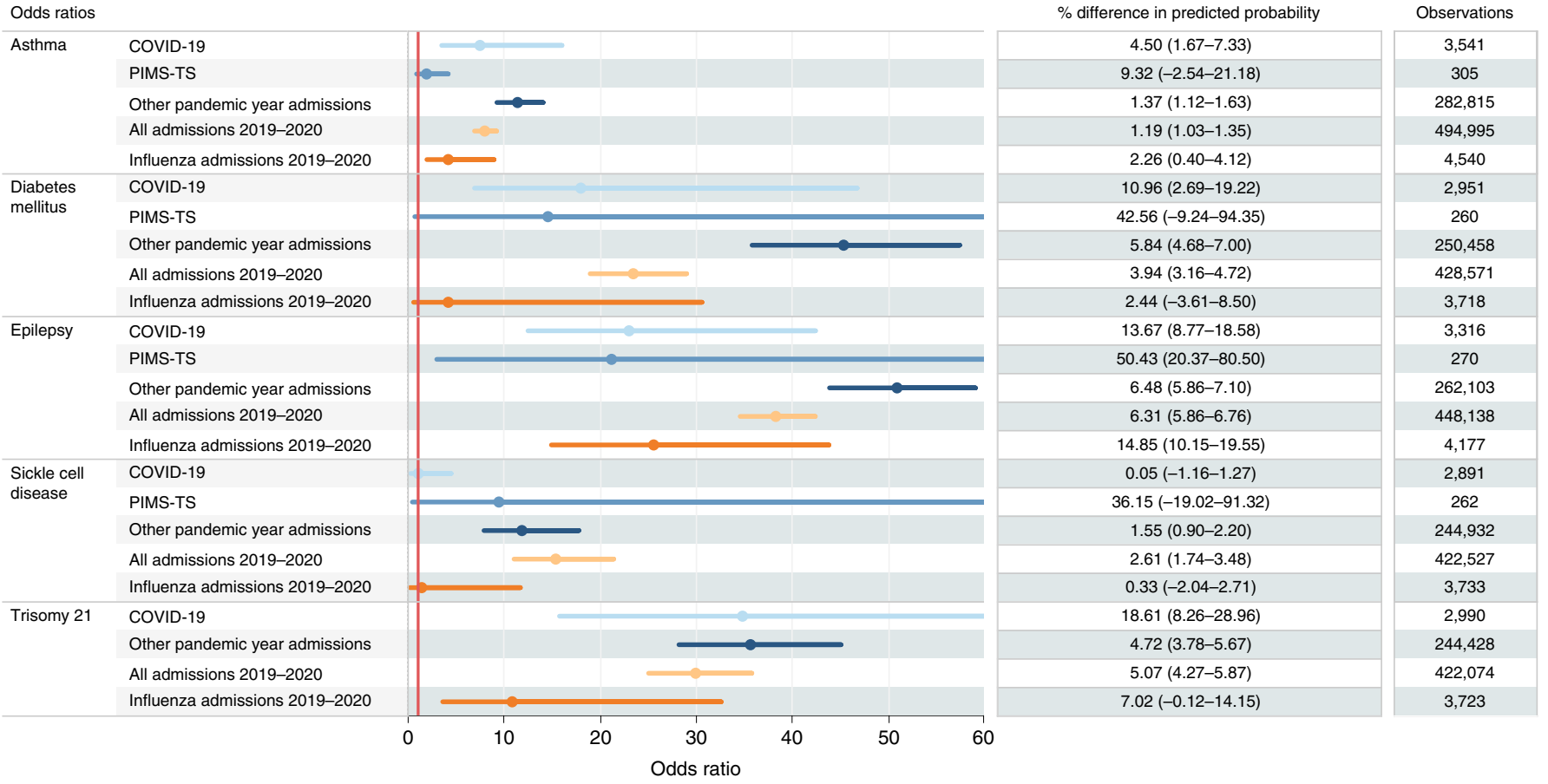

Fig. 4 | Odds ratios and predicted probability with $95 \%$ confidence intervals for admission to a PICU by selected diagnoses within each cohort, adjusted for age, sex, IMD category and ethnicity. Output from GEE models. Each mark and line represents odds ratios with $95 \%$ confidence intervals of PICU admission, within each cohort, among CYP with each comorbidity group compared to those without any comorbidity, adjusted for age, sex, IMD category and ethnicity. Percentage point difference in predicted probability of PICU admission with 95\% confidence intervals are shown in the middle panel. The number of observations in each model is shown in the right panel. These results are available in full in Supplementary Material 2.

primary care. Linking SUS data with national primary care records would improve identification of pre-existing conditions for these analyses, but these data are currently not available. Our analysis of individual or body system comorbidities does not account for CYP with both the comorbidity of interest and other conditions. However, we do assess odds of PICU admission by number of body systems involved, which does address identifying CYP with multiple medical problems. Finally, owing to incomplete coding, we were unable to examine some important risk factors for severe disease in adults in these analyses, including obesity ${ }^{24}$, which should be the focus of future study.

In conclusion, in marked contrast to adults, CYP were at very low risk of severe disease and death from COVID-19 or PIMS-TS during the first pandemic year. In the rare instances when CYP did require hospitalization, risk factors for severe disease were similar to those reported for adults. Additionally, the pattern of comorbidities was similar to that seen with influenza and all admissions in 2019-2020, reflecting underlying vulnerabilities to infection, although COVID-19 magnified these risks to a small degree. We identified important demographic factors that were associated with PICU admission due to PIMS-TS, although associations between comorbidities and PICU admission in this group were difficult to interpret.

\section{Online content}

Any methods, additional references, Nature Research reporting summaries, source data, extended data, supplementary information, acknowledgements, peer review information; details of author contributions and competing interests; and statements of data and code availability are available at https://doi.org/10.1038/ s41591-021-01627-9.

Received: 20 July 2021; Accepted: 11 November 2021; Published online: 20 December 2021

\section{References}

1. Verity, R. et al. Estimates of the severity of coronavirus disease 2019: a model-based analysis. Lancet Infect. Dis. 20, 669-677 (2020).

2. Swann, O. V. et al. Clinical characteristics of children and young people admitted to hospital with covid-19 in United Kingdom: prospective multicentre observational cohort study. BMJ 370, m3249 (2020).

3. Götzinger, F. et al. COVID-19 in children and adolescents in Europe: a multinational, multicentre cohort study. Lancet Child Adolesc. Health 4, 653-661 (2020).

4. Davies, N. G. et al. Age-dependent effects in the transmission and control of COVID-19 epidemics. Nat. Med. 26, 1205-1211 (2020).

5. de Siqueira Alves Lopes, A. et al. Coronavirus disease-19 deaths among children and adolescents in an area of Northeast, Brazil: why so many? Trop. Med. Int. Health 26, 115-119 (2021).

6. Ahmed, M. et al. Multisystem inflammatory syndrome in children: a systematic review. EClinicalMedicine 26, 100527 (2020).

7. Bhopal, S. S., Bagaria, J., Olabi, B. \& Bhopal, R. Children and young people remain at low risk of COVID-19 mortality. Lancet Child Adolesc. Health 5, e12-e13 (2021).

8. Feldstein, L. R. et al. Multisystem inflammatory syndrome in US children and adolescents. N. Engl. J. Med. 383, 334-346 (2020).

9. McCormick, D. W. et al. Deaths in children and adolescents associated with COVID-19 and MIS-C in the United States. Pediatrics 148, e2021052273 (2021).

10. Royal College of Paediatrics and Child Health. COVID-19-guidance on clinically extremely vulnerable children and young people. https://www.rcpch. ac.uk/resources/covid-19-guidance-clinically-extremely-vulnerable-childrenyoung-people (2021).

11. Song, X. et al. Comparison of clinical features of COVID-19 vs seasonal influenza A and B in US children. JAMA Netw. Open 3, e2020495 (2020).

12. Kompaniyets, L. et al. Underlying medical conditions associated with severe COVID-19 illness among children. JAMA Netw. Open 4, e2111182 (2021).

13. Harwood, R. et al. Which children and young people are at higher risk of severe disease and death after SARS-CoV-2 infection: a systematic review and individual patient meta-analysis. Preprint at https://www.medrxiv.org/content /10.1101/2021.06.30.21259763v1 (2021).

14. Davies, P. et al. Intensive care admissions of children with paediatric inflammatory multisystem syndrome temporally associated with SARS-CoV-2 (PIMS-TS) in the UK: a multicentre observational study. Lancet Child Adolesc. Health 4, 669-677 (2020). 
15. Flood, J. et al. Paediatric multisystem inflammatory syndrome temporally associated with SARS-CoV-2 (PIMS-TS): prospective, national surveillance, United Kingdom and Ireland, 2020. Lancet Regional Health Eur. 3, 100075 (2021)

16. Belay, E. D. et al. Trends in geographic and temporal distribution of US children with multisystem inflammatory syndrome during the COVID-19 pandemic. JAMA Pediatrics 175, 837-845 (2021).

17. Mathur, R. et al. Ethnic differences in SARS-CoV-2 infection and COVID19-related hospitalisation, intensive care unit admission, and death in 17 million adults in England: an observational cohort study using the OpenSAFELY platform. Lancet 397, 1711-1724 (2021).

18. Williamson, E. J. et al. Factors associated with COVID-19-related death using OpenSAFELY. Nature 584, 430-436 (2020).

19. Bhaskaran, K. et al. Factors associated with deaths due to COVID-19 versus other causes: population-based cohort analysis of UK primary care data and linked national death registrations within the OpenSAFELY platform. Lancet Reg. Health Eur. 6, 100109-100109 (2021).

20. Kanthimathinathan, H. K. et al. In the eye of the storm: impact of COVID-19 pandemic on admission patterns to paediatric intensive care units in the UK and Eire. Crit. Care 25, 1-9 (2021).
21. Docherty, A. B. et al. Features of 16,749 hospitalised UK patients with COVID-19 using the ISARIC WHO Clinical Characterisation Protocol. Preprint at https://www.medrxiv.org/content/10.1101/2020.04.23.20076042v1 (2020).

22. National Centre for Immunisation and Surveillance. COVID-19 in schools and early childhood education and care services-the experience in NSW: 16 June to 31 July 2021. https://www.ncirs.org.au/sites/default/files/2021-09/ NCIRS\%20NSW\%20Schools\%20COVID_Summary_8\%20September\%20 21_Final.pdf (2021)

23. Smith, C. et al. Deaths in children and young people in England after SARS-CoV-2 infection during the first pandemic year. Nat. Med. https://doi. org/10.1038/s41591-021-01578-1 (2021).

24. Yates, T. et al. Obesity, ethnicity, and risk of critical care, mechanical ventilation, and mortality in patients admitted to hospital with COVID-19: analysis of the ISARIC CCP-UK Cohort. Obesity (Silver Spring) 29, 1223-1230 (2021).

Publisher's note Springer Nature remains neutral with regard to jurisdictional claims in published maps and institutional affiliations.

(c) The Author(s), under exclusive licence to Springer Nature America, Inc. 2021 


\section{Methods}

Data. We used SUS, an administrative national database covering approximately $98 \%$ of National Health Service (NHS) hospital activity in England ${ }^{25}$. Data were available for admissions due to any cause in CYP aged 0-17 years in England between 1 March 2015 and 28 February $2021(n=11,467,027)$. Note that this does not include accident and emergency attendances not resulting in hospital admission. Coded fields included reason for hospital admission, comorbidities and sociodemographic characteristics. We primarily examined admissions occurring from 1 February 2019 to 31 January 2021, but, to account for variable quality and completeness of coding in SUS, we used all available data (2015-2021) from all types of admission (emergency, elective and maternal) to populate sociodemographic and comorbidity data for CYP.

As clinical details are limited in SUS, and to aid identification of COVID-19 and PIMS-TS admissions, these data were deterministically linked using unique patient NHS numbers to the following healthcare datasets:

1. Paediatric Intensive Care Audit Network (PICANet) data containing all PICU admissions in England

2. Death registrations provided by the Office for National Statistics (ONS)

3. National Child Mortality Database (NCMD), which collects preliminary notification data within $48 \mathrm{~h}$ of death of a CYP in England and Wales

4. SARS-CoV-2 PCR testing data provided by Public Health England (PHE)

Outcomes and exposures. We examined associations between severity outcomes (PICU admission or death) and the following exposures: reason for hospital admission (COVID-19, PIMS-TS or other), sociodemographic factors and presence of comorbidities.

We linked hospitalizations in SUS to PICANet data if the PICU admission date occurred during or within $1 \mathrm{~d}$ of the SUS admission or discharge date, to account for coding error in either dataset. We defined admissions resulting in death as the last admission for each CYP that occured within $28 \mathrm{~d}$ of death identified through ONS or NCMD. All NCMD deaths during the pandemic were clinically reviewed as part of a separate analysis ${ }^{23}$ to identify the contribution of SARS-CoV-2 infection and PIMS-TS to death.

Reason for admission. We used primary and secondary diagnoses coded to ICD-10 to define all hospital admissions between 1 February 2019 and 31 January 2021. We excluded all traumatic admissions (where the primary cause of admission was an external cause in ICD-10) and non-emergency admissions (that is, elective or maternity/newborn) from the analysis and classified the remainder into five cohorts:

- Admissions due to COVID-19 (1 February 2020 to 31 January 2021)

- Admissions due to PIMS-TS (1 February 2020 to 31 January 2021)

- All other admissions during the pandemic year (1 February 2020 to 31 January 2021)

- All admissions in the year before the pandemic (1 February 2019 to 31 January 2020)

- All admissions where the primary diagnosis was influenza in the year before the pandemic (1 February 2019 to 31 January 2020)

We defined COVID-19 admissions as those occurring after 1 February 2020 with relevant ICD-10 codes recorded as reason for admission or (using linked data) where there was a positive PCR test for SARS-CoV-2 within $7 \mathrm{~d}$ of admission or discharge (unless this occurred at least $7 \mathrm{~d}$ after admission to a PICU and nosocomial infection was likely).

We defined PIMS-TS admissions as those occurring after 1 February 2020 with ICD-10 codes recorded as reason for admission for either PIMS-TS (introduced in November 2020) or Kawasaki disease or systemic inflammatory response syndrome (used as proxies for PIMS-TS before November 2020).

To improve the identification of COVID-19 and PIMS-TS, we also reviewed details of all PICU admissions during the first pandemic year held within PICANet. Where treating specialists determined that the PICU admission was due to either COVID-19 or PIMS-TS, we recoded the SUS admission accordingly. Hospital admissions identified as both due to COVID-19 and PIMS-TS were defined as being due to PIMS-TS, as we assumed the COVID-19 diagnosis was part of the same disease process.

Sociodemographic exposures. Age was categorized as neonates (admission within month of birth), post-natal infants (admission between 1 and 11 months of birth), 1-4 years, 5-9 years, 10-14 years and 15-17 years. We defined ethnicity as White, Mixed, Asian, Black, Other and Unknown. We used IMD 2019 quintile categories to define the area-level socioeconomic status of CYP. Additional details of how IMD is defined are available in Supplementary Material 1.

Comorbidities. We used published literature and guidance on shielding to identify comorbidities likely to increase risk of severe SARS-CoV-2 disease $\mathrm{e}^{10,13}$. We identified CYP with chronic medical conditions by body system, those with life-limiting conditions and those with asthma, diabetes, epilepsy, sickle cell disease or trisomy 21 , using recognized ICD-10 code lists ${ }^{26,27}$. Note that admissions among CYP with specific conditions were also included in the broader body system diagnostic categories. We then defined additional comorbidity groups to examine vulnerability associated with multiple medical problems. These were defined as follows: comorbidities in more than one body system; and comorbidities in both neurological and respiratory, neurological and cardiovascular or respiratory and cardiovascular body systems. We compared admissions among CYP with each comorbidity category to CYP with no comorbidities in any category in all analyses.

Statistics and reproducibility. First, we described the characteristics of each of the five cohorts: admissions due to COVID-19, admissions due to PIMS-TS, other non-traumatic admissions in 2020-2021, all non-traumatic admissions in 20192020 and admissions due to influenza in 2019-2020. We supressed cell counts with small numbers (where $n<5$ ) due to the risk of identification of individuals, in line with guidance from data providers used in this study.

We then modeled the association between sociodemographic factors and comorbidities with PICU admission within each cohort separately. Sample sizes for these analyses were determined by the number of admissions identified in the SUS data, after non-emergency admissions and those due to trauma were excluded. Investigators were not blinded, and experiments were not randomized. All analyses were performed in Stata 16 (StataCorp). Models employed generalized estimation equations (GEEs) using the xtgee command to account for multiple admissions within the same CYP across and within different cohorts. Models used a logit link, specifying the covariance structure as 'exchangeable' (that is, we assumed equal correlations between any two admissions within one CYP). We then calculated the difference in predicted probability for PICU admission among those with and without each comorbidity category using the margins post-estimation command. We used univariable and then multivariable models to estimate the odds of PICU admission within each cohort by the presence of specific comorbidities compared to CYP with no comorbidities across any diagnostic category (dichotomous or ordinal variable), adjusted for age group (categorized as neonate, post-neontal infant, 1-4 years, 5-9 years, 10-14 years and 15-17 years); sex; ethnic group (categorized as White, Mixed, Asian, Black,Other and uknown); and IMD category (categorized as lowest to highest quintile category). Comparisons between cohorts were not tested, and significance was inferred if $95 \%$ confidence intervals did not overlap. We were unable to model death as an outcome in these analyses due to low numbers. In sensitivity analyses, we repeated analyses to include only secondaryschool-aged CYP (that is, ages 11-17 years) to inform vaccination policy.

Ethics approval and legal basis for data linkage and analyses. Ethics approval was provided after review by Yorkshire and the Humber, South Yorkshire NHS Research Ethics Committee on 10 June 2021 (reference: 21/YH/0127).

Informed consent was not obtained to use hospital administrative data for research purposes. Patients have the ability to opt out of having their personal/ confidential information being shared by NHS Digital and PHE and all other health and care organizations included in this analysis, for purposes not related to their own direct care. Additional information regarding the national opt-out can be found at https://www.nhs.uk/your-nhs-data-matters/manageyour-choice/. Current Control of Patient Information regulations provide a legal basis for linking datasets used in this study without consent ${ }^{28}$. Low numbers $(n<5)$ are suppressed to reduce the risk of identifying patients.

NCMD. The NCMD legal basis to collect confidential and personal-level data under the Common Law Duty of Confidentiality was established through the Children Act 2004, Sections M and N; Working Together to Safeguard Children 2018 (https://consult.education.gov.uk/child-protection-safeguarding-and-familylaw/working-together-to-safeguard-children-revisions-t/supporting_documents/ Working\%20Together\%20to\%20Safeguard\%20Children.pdf); and associated Child Death Review Statutory \& Operational Guidance (https://assets.publishing. service.gov.uk/government/uploads/system/uploads/attachment_data/file/859302/ child-death-review-statutory-and-operational-guidance-england.pdf). The NCMD legal basis to collect personal data under the General Data Protection Regulation (GDPR) without consent is defined by GDPR Article 6 (e) Public task and by GDPR Article 9 (h) Health or social care (with a basis in law).

PICANet. Processing of personally identifiable data for the purposes of service evaluation, audit and research was approved by the Patient Information Advisory Group (now the Health Research Authority Confidentiality Advisory Group) in 2002 under Section 60 of the Health and Social Care Act (subsequently Section 251 of the National Health Service Act 2006) (reference: PIAG 4-07(c) 2002). Permissions to use these data were amended and approved specifically to collect additional data relating to COVID-19 for confirmed and suspected cases.

Reporting Summary. Further information on research design is available in the Nature Research Reporting Summary linked to this article.

\section{Data availability}

These analyses were undertaken using datasets held by NHS England for the use of ongoing service evaluation, held within the National Commissioning Data Repository. Access to these data at the individual level is restricted, as described in data sharing agreements between NHS England and specific data providers and 
within the application for ethics approval provided for this study. We were able to access and analyze these data as employees of NHS England. Researchers who want to access the individual-level data used in this analysis may apply to do so via NHS Digital. Aggregated, non-identifiable data used for this study are provided in the Supplementary Material.

\section{References}

25. Boyd A. et al. Understanding Hospital Episode Statistics (HES). https://www. closer.ac.uk/wp-content/uploads/CLOSER-resource-understanding-hospitalepisode-statistics-2018.pdf (2017).

26. Hardelid, P., Dattani, N. \& Gilbert, R. Estimating the prevalence of chronic conditions in children who die in England, Scotland and Wales: a data linkage cohort study. BMJ Open 4, e005331 (2014).

27. Fraser, L. K. et al. Rising national prevalence of life-limiting conditions in children in England. Pediatrics 129, e923-e929 (2012).

28. NHS Digital. Control of patient information (COPI) notice. https://digital nhs.uk/coronavirus/coronavirus-covid-19-response-informationgovernance-hub/control-of-patient-information-copi-notice (2021).

\section{Acknowledgements}

We would like to thank the National Child Mortality Database; the Paediatric Intensive Care Audit Network; Public Health England; NHS Digital; NHS England; and the NHS Improvement Children and Young People Team, particularly R. Owen, S. Solti, T. Watson-Koszel, M. Ambia, C. Styles and A. Wojciechowska, for their support in identifying, linking and making the data used in this study available for analysis. J.L.W. is in receipt of a Medical Research Council fellowship (grant no. MR/R00160X/1). R.H is in receipt of a fellowship from Kidney Research UK (grant no. TF_010_20171124).
L.K.F. is in receipt of funding from Martin House Children's Hospice (there is no specific grant number for this). R.M.V. is in receipt of a grant from the National Institute of Health Research to support this work (grant no. NIHR202322). D.H. is supported by the National Institute for Health Research through the National School for Public Health Research Programme and the Applied Health Research Programme for North West London. Funders had no role in study design, data collection, analysis, decision to publish or preparation of the manuscript.

\section{Author contributions}

The study design was developed by all authors. Data cleaning and analysis was performed by J.L.W., L.K.F. and R.M.V. Data interpretation was performed by all authors. The first draft was written by J.L.W. All authors contributed to editing and reviewing the final manuscript.

\section{Competing interests}

The authors declare no competing interests.

\section{Additional information}

Supplementary information The online version contains supplementary material available at https://doi.org/10.1038/s41591-021-01627-9.

Correspondence and requests for materials should be addressed to Joseph L. Ward.

Peer review information Nature Medicine thanks the anonymous reviewers for their contribution to the peer review of this work. Alison Farrell and João Monteiro are the primary editors on this article and managed its editorial process and peer review in collaboration with the rest of the editorial team.

Reprints and permissions information is available at www.nature.com/reprints. 


\section{Reporting Summary}

Nature Research wishes to improve the reproducibility of the work that we publish. This form provides structure for consistency and transparency in reporting. For further information on Nature Research policies, see our Editorial Policies and the Editorial Policy Checklist.

\section{Statistics}

For all statistical analyses, confirm that the following items are present in the figure legend, table legend, main text, or Methods section.

$\mathrm{n} / \mathrm{a}$ Confirmed

$\bigotimes$ The exact sample size $(n)$ for each experimental group/condition, given as a discrete number and unit of measurement

\ A statement on whether measurements were taken from distinct samples or whether the same sample was measured repeatedly

The statistical test(s) used AND whether they are one- or two-sided

Only common tests should be described solely by name; describe more complex techniques in the Methods section.

$\bigotimes$ A description of all covariates tested

\ A description of any assumptions or corrections, such as tests of normality and adjustment for multiple comparisons

$\triangle$ A full description of the statistical parameters including central tendency (e.g. means) or other basic estimates (e.g. regression coefficient)

AND variation (e.g. standard deviation) or associated estimates of uncertainty (e.g. confidence intervals)

For null hypothesis testing, the test statistic (e.g. $F, t, r$ ) with confidence intervals, effect sizes, degrees of freedom and $P$ value noted Give $P$ values as exact values whenever suitable.

Х $\square$ For Bayesian analysis, information on the choice of priors and Markov chain Monte Carlo settings

$\square$ For hierarchical and complex designs, identification of the appropriate level for tests and full reporting of outcomes

Х $\square$ Estimates of effect sizes (e.g. Cohen's $d$, Pearson's $r$ ), indicating how they were calculated

\section{Our web collection on statistics for biologists contains articles on many of the points above.}

\section{Software and code}

Policy information about availability of computer code

Data collection Data collection was performed for routine national administrative purposes. No software was used for data collection related to this analysis.

Data analysis All analyses were performed in Stata 16 (StataCorp, College Station TX).

For manuscripts utilizing custom algorithms or software that are central to the research but not yet described in published literature, software must be made available to editors and

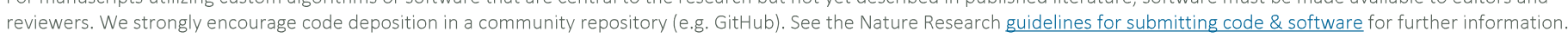

\section{Data}

Policy information about availability of data

All manuscripts must include a data availability statement. This statement should provide the following information, where applicable:

- Accession codes, unique identifiers, or web links for publicly available datasets

- A list of figures that have associated raw data

- A description of any restrictions on data availability

These analyses were undertaken using datasets held by NHS England for the use of ongoing service evaluation, stored within the National Commissioning Data Repository. Access to these data at individual level are restricted, as described in data sharing agreements between NHS England and specific data providers, and within in the application for ethical approval provided for this study. Aggregated, non-identifiable data used for this study are provided in the supplementary material. 
Please select the one below that is the best fit for your research. If you are not sure, read the appropriate sections before making your selection.

$\bigotimes$ Life sciences $\quad \square$ Behavioural \& social sciences $\quad \square$ Ecological, evolutionary \& environmental sciences

For a reference copy of the document with all sections, see nature.com/documents/nr-reporting-summary-flat.pdf

\section{Life sciences study design}

All studies must disclose on these points even when the disclosure is negative.

$\begin{array}{ll}\text { Sample size } & \begin{array}{l}\text { 11 million admissions. Datasets were included in order to } \\ \text { 1) Analyse all admissions amongst children and young people in England during the pandemic year and years prior for comparison (Secondary } \\ \text { Use Service Data) } \\ \text { 2) Analyse all Paediatric Intensive Care unit admissions and deaths in England (PICANeT, National Child Mortality Database, Office for National } \\ \text { Statistics Death Registrations) to understand clinical outcomes after admission with COVID-19 and other causes, } \\ \text { 3) Analyse all positive PCR tests for SARS-CoV-2 in England during the pandemic linked to admission data in order to identify admissions } \\ \text { related to COVID-19. }\end{array} \\ \text { Data exclusions } & \begin{array}{l}\text { Analyses were restricted to emergency non-traumatic admissions from Feb 1st 2019- Jan 31st 2021. However, all available data were } \\ \text { included for the purposes of populating co-morbidity and sociodemographic fields }\end{array} \\ \text { Replication } & \text { Not applicable. Observational study design of hospital administrative data } \\ \text { Randomization Not applicable. Observational study design of hospital administrative data } & \text { Not applicable. Observational study design of hospital administrative data } \\ \text { Blinding } & \text { Notal }\end{array}$

\section{Reporting for specific materials, systems and methods}

We require information from authors about some types of materials, experimental systems and methods used in many studies. Here, indicate whether each material, system or method listed is relevant to your study. If you are not sure if a list item applies to your research, read the appropriate section before selecting a response.

\begin{tabular}{|c|c|c|c|}
\hline \multicolumn{2}{|r|}{ Materials \& experimental systems } & \multicolumn{2}{|c|}{ Methods } \\
\hline$n / a$ & Involved in the study & $n / a$ & Involved in the study \\
\hline$\bigotimes$ & $\square$ Antibodies & Х & $\square$ ChIP-seq \\
\hline Х & Eukaryotic cell lines & Х & $\square$ Flow cytometry \\
\hline$\bigotimes$ & $\square$ Palaeontology and archaeology & Х & $\square$ MRI-based neuroimaging \\
\hline Х & $\square$ Animals and other organisms & & \\
\hline$\square$ & $\bigotimes$ Human research participants & & \\
\hline$\bigotimes$ & $\square$ Clinical data & & \\
\hline$\bigotimes$ & $\square$ Dual use research of concern & & \\
\hline
\end{tabular}

\section{Human research participants}

\section{Policy information about studies involving human research participants}

Population characteristics

Recruitment

Ethics oversight
All children and young people aged 0-17 admitted to secondary care in England between March 2015 - Jan 31st 2021. Characteristics of this population (age, sex, ethnicity, deprivation, previous co-morbidities) are provided in tables 1 and 2 and within Supplementary Material

Participants were not recruited. Analyses were performed on hospital administrative data linked to other clinical datasets.

Ethics approval was provided after review by Yorkshire and the Humber, South Yorkshire NHS Research Ethics Committee on 10th June 2021 (Reference 21/YH/0127).

Current Control Of Patient Information (COPI) regulations provide a legal basis for linking these datasets without consent 